\title{
Comment on 'A diagnostic algorithm to distinguish desmoplastic from spindle cell melanoma'
}

Modern Pathology (2014) 27, 1421; doi:10.1038/modpathol.2014.52

To the Editor: I read with interest the article by Weissinger et $a l^{1}$ 'A diagnostic algorithm to distinguish desmoplastic from spindle cell melanoma' published in the September 2013 issue. They propose MelanA as a first and, if positive, exclusionary step in their algorithm. This appears to be based on their cluster analysis of 38 cases (16 spindle cell, 18 desmoplastic, 4 mixed). All 4 of their mixed and all 18 of their desmoplastic melanomas were negative for MelanA. Their meta-review (Supplementary Table 9) is a summary of published immunoprofiles comparing spindle cell to desmoplastic melanoma. It is based on much larger number of cases and suggests that MelanA is not quite that specific and that HMB-45 may actually be of superior discriminatory value than MelanA.

\begin{tabular}{lrrrrrrrr}
\hline & \multicolumn{3}{c}{ Spindle cell } & & \multicolumn{3}{c}{ Desmoplastic } \\
\cline { 2 - 3 } \cline { 7 - 9 } & + & tested & $\%$ & & + & tested & $\%$ \\
\hline HMB-45 & 54 & 118 & 45.8 & 49 & 559 & 8.8 \\
MelanA/MART-1 & 29 & 66 & 43.9 & & 44 & 234 & 18.8 \\
\hline
\end{tabular}

A comment on the difference between their cluster analysis and meta-review of published literature in this regard would be appreciated.

\section{Disclosure/conflict of interest}

The author declares no conflict of interest.

$$
\begin{array}{r}
\text { Theodore M Parsons } \\
{ }^{1} \\
\text { Department of Pathology, Anatomy and Cell } \\
\text { Biology, Thomas Jefferson University, } \\
\text { Philadelphia, PA, USA } \\
\text { E-mail: theodore.parsons@jefferson.edu }
\end{array}
$$

\section{References}

1 Weissinger SE, Keil P, Silvers DN, et al. A diagnostic algorithm to distinguish desmoplastic from spindle cell melanoma. Mod Pathol 2014;27:524-534.

\section{Comparison of MelanA/MART-1 and HMB45 labeling in desmoplastic melanoma}

Modern Pathology (2014) 27, 1421-1423; doi:10.1038/modpathol.2014.59

To the Editor: We thank Dr Parsons ${ }^{1}$ for his comment and would like to clarify our decision to include and prefer MelanA/MART-1 in our diagnostic algorithm to distinguish spindle cell from desmoplastic melanoma. ${ }^{2}$

The summary of our literature meta-review that Dr Parsons refers to tabulates the MelanA/MART-1 and HMB45 labeling fraction in spindle cell and desmoplastic melanoma derived from 67 different studies. The apparent difference of marker fractions in desmoplastic melanoma (8.8 vs 18.8\%; $P<0.0001 ; n=54$ studies) derives in large parts from studies $(n=37)$ where only one of the two biomarkers was assessed. The key limitation of this approach to summarize data for the assessment of discriminatory value is, that the percentage of positive cases is based on a theoretical summary of separate studies on different cases rather than a direct comparison of two markers in the same samples. In other words, we do not know the MelanA/MART-1 status in the $\sim 300$ more desmoplastic melanomas that have only been stained for HMB45 (but not for MelanA/MART-1; ie, discrepant analysis). To assess and compare the discriminatory value of these two biomarkers, it is crucial to restrict the comparison to studies where samples have been assessed with both markers (Figure 1a). When restricting the tabulation to those nine studies that allow analysis of both markers on a case-by-case basis (Figure 1b), ${ }^{2-11}$ there is no significant difference in the labeling fraction of HMB45 and MelanA/MART-1 in desmoplastic melanoma $(P=0.79)$. 